\title{
Lesão renal aguda: assistência de enfermagem durante a sessão de hemodiálise em
}

\section{Unidade de Terapia Intensiva}

\author{
Acute kidney injury: nursing care during the hemodialysis session in an Intensive Care Unit \\ Lesión renal aguda: cuidados de enfermería durante la sesión de hemodiálisis en una Unidad de

Valdjane Nogueira Noleto Nobre ORCID: https://orcid.org/0000-0002-3831-5003 Universidade Federal de Minas Gerais, Brasil

E-mail: valdjane.nobre@ebserh.gov.br

Pamela Nery do Lago

ORCID: https://orcid.org/0000-0002-3421-1346

Universidade Federal de Minas Gerais, Brasil

E-mail:pamela.lago@ebserh.gov.br

Erlon Carlos Vieira

ORCID: https://orcid.org/0000-0002-8837-5274

Universidade Federal de Minas Gerais, Brasil

E-mail: erlon.vieira@ebserh.gov.br

Marlene Simões e Silva

ORCID: https://orcid.org/0000-0002-1195-493X

Universidade Federal de Minas Gerais, Brasil

E-mail:marlene.simoes@ebserh.gov.br

Flávia Cristina Duarte Silva

ORCID: https://orcid.org/0000-0002-5271-7172

Universidade Federal de Minas Gerais, Brasil

E-mail: flavia.duarte@ebserh.gov.br

Ronaldo Antônio de Abreu Junior

ORCID: https://orcid.org/0000-0001-9568-5644

Universidade Federal de Minas Gerais, Brasil

E-mail: ronaldo.abreu@ebserh.gov.br

Roseane Pereira Sousa

ORCID: https://orcid.org/0000-0002-0928-6637

Universidade Federal de Minas Gerais, Brasil

E-mail: roseane.pereira@ebserh.gov.br

Andrea Aparecida Martins de Carvalho

ORCID: https://orcid.org/0000-0003-3355-9735

Universidade Federal de Minas Gerais, Brasil

E-mail: princaandreia@gmail.com

Martapolyana Torres Menezes da Silva

ORCID: https://orcid.org/0000-0002-9517-2600

Universidade Federal do Rio Grande do Norte, Brasil

E-mail: martapolyana.silva@ebserh.gov.br

Gisela Pereira Xavier Albuquerque

ORCID: https://orcid.org/0000-0002-9712-2236 Universidade Federal do Pará, Brasil E-mail: gisela.xavier@ebserh.gov.br

Rosiana Lima Prado

ORCID: https://orcid.org/0000-0003-0403-7188 Universidade Federal de Sergipe, Brasil E-mail: rosiana.prado@ebserh.gov.br

Andrea Paula Dourado Vasconcelos ORCID: https://orcid.org/0000-0002-3619-4754

Hospital Universitário de Pernambuco, Brasil E-mail: andreapdourado@hotmail.com

Ana Luiza Menezes Santos

ORCID: https://orcid.org/0000-0001-7310-4036

Universidade Federal de Sergipe, Brasil

E-mail: analuiza.huufs@ebserh.gov.br

Fabiana Nascimento Silva

ORCID: https://orcid.org/0000-0001-6201-6305

Universidade Federal de Sergipe, Brasil

E-mail: fabiana.nascimento@ebserh.gov.br 
Francisco Rodrigues Martins

\begin{abstract}
Resumo
A lesão renal aguda é uma síndrome clínica reversível com perda abrupta da função renal em que apresenta redução do ritmo de filtração glomerular e/ou oligúria. É um grave problema nas unidades de terapia intensiva e consequentemente para a saúde pública, devido elevado índice de óbito e alto custo do tratamento. O presente estudo objetivou analisar e descrever as intervenções de enfermagem frente a paciente com lesão renal aguda nas principais intercorrências no decurso da sessão de hemodiálise em unidade de terapia intensiva. Trata-se de um estudo de revisão bibliográfica do tipo narrativa e de cunho descritivo qualitativo, extraídos de artigos científicos nas bases de dados: BVS, LILACS, BIREME, SCIELO e PUBMED, Sociedade Brasileira de Nefrologia, livros e Resolução da Diretoria Colegiada que aborda a lesão renal, terapia renal substitutiva e unidade de terapia intensiva. Como resultado tem-se que fatores de riscos são as comorbidades, além da sepse, idade elevada, choque hipovolêmico, nefrotoxicidade, nódulos, isquemia e internação prolongada nas unidades de terapia intensiva. Conclui-se que o papel do enfermeiro é primordial e indispensável para identificar, monitorar e intervir nas complicações durante a hemodiálise. Contudo ainda se faz necessário maiores estudos sobre a enfermagem como membro da equipe multiprofissional e sua importância no plano de cuidados destes pacientes.
\end{abstract}

Palavras-chave: Lesão renal; Diálise renal; Unidade de terapia intensiva; Cuidados de enfermagem.

\begin{abstract}
Acute kidney injury is a reversible clinical syndrome with abrupt loss of kidney function in which there is a reduction in the glomerular filtration rate and/or oliguria. It is a serious problem in intensive care units and consequently for public health, due to the high death rate and high cost of treatment. This study aimed to analyze and describe the nursing interventions for patients with acute kidney injury in the main complications during the hemodialysis session in an intensive care unit. This is a literature review study of the narrative type and qualitative descriptive nature, extracted from scientific articles in the databases: BVS, LILACS, BIREME, SCIELO and PUBMED, Brazilian Society of Nephrology, books and Resolution of the Collegiate Board that addresses renal injury, renal replacement therapy and intensive care unit. As a result, risk factors are comorbidities, in addition to sepsis, older age, hypovolemic shock, nephrotoxicity, nodules, ischemia and prolonged hospitalization in intensive care units. It is concluded that the role of the nurse is essential and essential to identify, monitor and intervene in complications during hemodialysis. However, further studies are needed on nursing as a member of the multidisciplinary team and its importance in the care plan for these patients.
\end{abstract}

Keywords: Kidney damage; Renal dialysis; Intensive care unit; Nursing care.

\title{
Resumen
}

La lesión renal aguda es un síndrome clínico reversible con pérdida abrupta de la función renal en el que hay una reducción de la tasa de filtración glomerular y / u oliguria. Es un problema grave en las unidades de cuidados intensivos y en consecuencia para la salud pública, debido a la alta tasa de mortalidad y el alto costo del tratamiento. Este estudio tuvo como objetivo analizar y describir las intervenciones de enfermería a pacientes con daño renal agudo en las principales complicaciones durante la sesión de hemodiálisis en una unidad de cuidados intensivos. Se trata de un estudio de revisión de la literatura de tipo narrativo y cualitativo descriptivo, extraído de artículos científicos en las bases de datos: BVS, LILACS, BIREME, SCIELO y PUBMED, Sociedad Brasileña de Nefrología, libros y Resolución de la Junta Colegiada que aborda la lesión renal, terapia de reemplazo renal y unidad de cuidados intensivos. Como resultado, los factores de riesgo son las comorbilidades, además de la sepsis, la vejez, el shock hipovolémico, la nefrotoxicidad, los nódulos, la isquemia y la hospitalización prolongada en unidades de cuidados intensivos. Se concluye que el rol de la enfermera es fundamental e imprescindible para identificar, monitorear e intervenir en las complicaciones durante la hemodiálisis. Sin embargo, son necesarios más estudios sobre la enfermería como miembro del equipo multidisciplinar y su importancia en el plan de cuidados de estos pacientes.

Palabras clave: Daño renal; Diálisis renal; Unidad de terapia intensiva; Cuidado de enfermera.

\section{Introdução}

A lesão renal aguda (LRA) é uma síndrome clínica reversível com perda rápida da função renal com redução do ritmo de filtração glomerular e/ou oligúria, é determinado por alteração da creatinina sérica e/ou débito urinário. É um problema relevante nas unidades de terapia intensiva (UTIs) e para a saúde pública, por causa do elevado índice de óbito e alto custo do tratamento (Schor, Durão Junior \& Kirsztajn, 2017). 
A LRA constitui índices mais elevados de morbimortalidade no Brasil e no mundo, considerado uma das principais causas de morte em UTI, quando não diagnosticado e tratado precocemente, evolui para o estágio mais crônicos da doença. Os números de óbitos ressaltam na urgência de medidas enérgicas e efetivas na tomada de decisão diante do paciente portador de LRA (Padilha, Vattimo, Silva \& Kimura, 2010).

Segundo Resolução $\mathrm{n}^{\circ} 7$ (2010) a UTI é um setor crítico e complexo destinado à internação e permanência de pacientes em estado grave, que exigem observação e monitoramento constante, estes são predispostos à falência de órgãos vitais como os rins que necessita de cuidado constante de profissionais especializados, materiais específicos e tecnologias necessárias ao diagnóstico, monitoramento e terapêutica adequada, onde o processo de trabalho possibilite assistência ininterrupta e integral.

Para construção deste artigo, evidencia-se a relevância da assistência de enfermagem durante a sessão de hemodiálise em portador de LRA na UTI, pois se justifica uma vez que a LRA é uma dais maiores causas de morbimortalidade e permanência prolongada nas UTIs. Neste sentido, o presente trabalho abordará esta temática tendo em vista medidas efetivas que objetivam aprimorar a qualidade da assistência de enfermagem visando promoção da saúde, prevenção de agravos, da disfunção orgânica e do óbito de forma organizada e sistematizada.

O presente estudo objetivo analisar e descrever as intervenções de enfermagem frente a paciente com lesão renal aguda nas principais intercorrências no decurso da sessão de hemodiálise em unidade de terapia intensiva.

Diante disto, no cenário da enfermagem faz-se indispensável à utilização da Sistematização da Assistência de Enfermagem (SAE), uma vez que a mesma possibilita ao enfermeiro prestar uma assistência com mais qualidade e eficiência, onde este método corrobora no sentido de planejar, orientar e organizar o processo de trabalho, tornando factível o registro da prática profissional, executando e documentando a assistência prestada, permitindo evidenciar a contribuição da enfermagem na atenção à saúde da população ampliando a visibilidade e o reconhecimento profissional.

Conforme a Resolução n 358 (2009), a SAE é um método utilizado no processo de trabalho que possibilita uma assistência de enfermagem integral e humanizada que deve ser colocado em prática em todo ambiente de saúde, seja ele público ou privado, trazendo respaldo científico ao profissional enfermeiro.

A intervenção de enfermagem faz parte do processo do cuidado prestando ao paciente e seus familiares, contribuindo no controle dos temores, intervindo e avaliando os resultados diante das medidas adotadas e se necessário modificando-as, com vistas à melhora do doente, possibilitando sua cura.

O estudo apresenta o conceito e classificação da LRA, as principais causas desta injúria em UTI e apresenta assistência de enfermagem frente ao paciente no tratamento hemodialítico. Nesta conjuntura, o presente estudo abordará a LRA devido à importância profissional em desenvolver medidas mais efetivas e ágeis que possam melhorar a assistência de enfermagem na perspectiva de prevenir complicações como a disfunção orgânica e morte dos pacientes portadores de LRA.

\section{Metodologia}

Trata-se de um estudo de revisão bibliográfica, do tipo narrativa e de cunho descritivo qualitativo (Marconi \& Lakatos, 2017) extraído dos principais bancos de dados da literatura. Para tanto foram realizadas pesquisas em artigos científicos sobre o tema no período de outubro 2020 a fevereiro 2021, utilizando os descritores: lesão renal, diálise renal, unidade de terapia intensiva e cuidados de enfermagem. Para construção do mesmo foi analisado e lido na íntegra 50 artigos científicos, sendo eleitos 24 de relevância para o desenvolvimento do tema.

Foi considerado como critério de inclusão artigos publicados no período de 2011 a 2020, que se encontravam na língua portuguesa, texto completos, Resolução da Diretoria Colegiada (RDC), em livros que se delimitasse a LRA para o desenvolvimento da pesquisa, retirados das bases de dados BVS (Biblioteca Virtual de Saúde), SciELO (Scientific Eletronic 
Library Oline), LILACS (Literatura Latino-americana e do Caribe em Ciências da Saúde) e ainda Google acadêmico.

Foram excluídas as literaturas com mais de 10 anos de publicação, artigos de revisão e os que não contribuíssem diretamente para pesquisa. Para seleção do material foram analisados e selecionados com base nos títulos e posteriormente nos resumos, visando analisar a assistência de enfermagem durante a sessão de hemodiálise em UTI. Por fim, realizada a análise dos dados coletados para o desenvolvimento do mesmo e elaboração das considerações finais acerca do presente estudo, instituindo consonância com os objetivos fundamentados (Marconi \& Lakatos, 2017).

\section{Resultados e Discussão}

A LRA constitui-se quando os rins (órgãos pares responsáveis pela homeostase do organismo) são impossibilitados por algum advento, extrair os produtos de deterioração metabólicos do organismo ou de realizar seu papel regulador. As substâncias normalmente extraídas na urina concentram-se nos líquidos corporais em decorrência da depleção da excreção renal, afetando as funções endócrinas e metabólicas (Smeltzer \& Bare, 2014).

Segundo Sousa (2015), os rins exercem funções fundamentais e indispensáveis na conservação do volume de líquido corporal e na constituição eletrolítica adequada no organismo humano para desenvolver bom desempenho de suas funções. Contudo, constantemente esse sistema apresenta desequilíbrio no funcionamento ao lidar com pacientes críticos, especialmente quando apresenta distúrbio renal, inclusive decorrente da LRA, levando a um aumento acentuado da mortalidade nas UTIs.

Para Pares, Wandeur e Matsuo (2013) a LRA é a supressão parcial ou total da função renal de forma súbita, causando redução da função renal e aumento considerado da uréia e creatinina, sendo associado ou não a oligúria, desta forma causa aos rins incapacidade de preservar o equilíbrio ácido-básico, desempenhar seu papel excretor e de manter o equilíbrio funcional do organismo.

Nesta conjuntura, para Li, Burdmann e Mehta (2013), a LRA é provocada por mecanismo que ocasiona a diminuição da taxa de filtração glomerular, de modo abrupto e que perdura por horas ou dias. Os fatores são diversos, com causas que pode ser extrarrenais ou sistêmica, classificando-se em pré-real, renal e pós renal. Nestas condições o rim fica impossibilitado de desempenhar seu papel fisiológico de filtrar e excretar escórias e toxinas, manter a homeostase hidroeletrolítico e o equilíbrio ácido-base do organismo.

Neste contexto Silva, Silva, Silva, Maia e Oliveira (2016), corrobora que os principais fatores além da idade são: nefrotoxicidade, infecções, nódulos, choque hipovolêmico, cardiogênico e séptico, isquemia, internamento prolongado nas UTIs, além de outros fatores com menor importância, porem com significância.

Para melhor exemplificação dos tipos de LRA segue Tabela 1 abaixo: 
Tabela 1 - Categorização da LRA.

\begin{tabular}{|c|c|c|}
\hline Tipo de LRA & Local do acometimento & Causas \\
\hline Pré-renal & $\begin{array}{l}\text { Ocasionada por hipoperfusão real, ou } \\
\text { seja, eventos que ocorre antes do rim. }\end{array}$ & $\begin{array}{l}\text { Em decorrente de causas sistêmicas como } \\
\text { hipovolemia por desidratação como, hemorragia, } \\
\text { vômito, diarreia e inclui ainda grandes queimaduras e } \\
\text { uso de diuréticos entre outros. }\end{array}$ \\
\hline Renal & $\begin{array}{l}\text { Compromete estruturas interna do rim, ou } \\
\text { seja, intrínseca ao próprio rim. }\end{array}$ & $\begin{array}{l}\text { São de origem isquêmica, toxidade ou sepse, estas } \\
\text { são as principais causas em pacientes graves. Aponta } \\
\text { que chega até } 60 \% \text { das ocorrências. }\end{array}$ \\
\hline Pós-renal & $\begin{array}{l}\text { Atinge estruturas das vias urinárias } \\
\text { posterior aos rins ao longo dos ureteres. } \\
\text { Podendo ser internamente ou } \\
\text { externamente. }\end{array}$ & $\begin{array}{l}\text { Por obstrução das vias urinárias, seja de origem } \\
\text { ureteral, vesical ou uretral, que pode ser } \\
\text { desencadeado por nódulos benignos ou malignos, } \\
\text { cálculos renais, distúrbios retroperitoneais ou } \\
\text { estenose entre outras causas. Representa-se em menor } \\
\text { prevalência com } 5 \text { a } 10 \% \text { dos casos. }\end{array}$ \\
\hline
\end{tabular}

Fonte: Schor et al. (2017).

Pode ser observado que a LRA é de três tipos e origem distintas, sendo intrínseca ou não ao rim, ocasionado principalmente por hipoperfusão renal, isquemia do parênquima renal, nefrotoxidade, obstrução ou decorrência de sepse, estas são as principais causas em pacientes hospitalizados, acentuando esta incidência em pacientes internados em UTI. É de grande valia destacar que a recuperação da função renal depende do tempo que leva para sanar o problema primário o qual desencadeou, independentemente do tipo da mesma. Se corrigido em tempo hábil os rins podem recuperar sua função mediante diagnóstico precoce e tratamento apropriado.

Para Nunes, Brunetta, Leal, Pisi e Roris Filho (2010) a lesão renal aguda é uma condição clínica que tem relação direta com a taxa de filtração glomerular (ureia e creatinina sérica) e débito urinário, desta forma desencadeia-se elevado número de casos de morbimortalidade em curto e longo prazo, além de oportunizar surgimento de doença renal crônica posteriormente. Para melhor manejo do quadro clínico do paciente é indispensável a identificação do estágio da LRA e, para identificar o grau de acometimento, lança-se mão de análise de exames laboratoriais. Para tanto segue Tabela 2 classificando-a em estágios: 
Tabela 2 - Definição e Classificação dos estágios da lesão renal aguda

\begin{tabular}{|c|l|c|}
\hline Estágios & \multicolumn{1}{|c|}{ Creatinina sérica } & Diurese \\
\hline Estágio 1 & $\begin{array}{l}\text { Acréscimo de } 0,3 \mathrm{mg} / \mathrm{dl} \text { ou aumento de } 150-200 \% \\
(1,5 \mathrm{a} 2 \text { vezes }) \text { do valor basal }\end{array}$ & $<0,5 \mathrm{ml} / \mathrm{Kg} / \mathrm{h}$ por 6 horas \\
\hline Estágio 2 & $\begin{array}{l}\text { Acréscimo }>\text { ou }+=200-300 \% \text { do }(>2-3 \mathrm{vezes}) \\
\text { valor basal }\end{array}$ & $<0,5 \mathrm{ml} / \mathrm{Kg} / \mathrm{h}$ por $>12$ horas \\
\hline Estágio 3 & $\begin{array}{l}\text { Aumento }>\text { ou }=300 \%(>3 \mathrm{vezes}) \text { do valor basal } \\
\text { ou creatinina sérica } \geq 4,0 \mathrm{mg} / \mathrm{dl} \text { com aumento } \\
\text { agudo de pelo menos } 0,5 \mathrm{mg} / \mathrm{dl})\end{array}$ & $\begin{array}{c}<0,3 \mathrm{ml} / \mathrm{Kg} / \mathrm{h} \text { por } 24 \text { horas ou anúria por } \\
12 \text { horas }\end{array}$ \\
\hline
\end{tabular}

Fonte: Schor et al. (2017).

Segundo Nunes et al., (2010) a LRA representa em média de 5\% das internações hospitalares e até 30\% quando hospitalizados em UTI. A necrose tubular aguda é responsável por mais de 50\% das lesões renais agudas em pacientes hospitalizados e mais de 76\% dos casos nos pacientes de UTI, onde a septicemia é o fator mais associado. A predominância da LRA varia de acordo com os marcadores de creatinina utilizados no diagnóstico e tem importante impacto na mortalidade.

Observa-se que a LRA é diagnosticada quando a doença já se encontra instalada, pois para identificação desta patologia além de dispor de uma avaliação criteriosa de uma equipe multiprofissional, lança-se mão de medidas auxiliares como exames laboratoriais para verificar a taxa de filtração glomerular (parâmetros de creatinina, ureia), ácido base, potássio entre outros, incluindo o monitoramento da diurese (Barros et al., 2012).

Foi identificado que a LRA avulta com a idade, sendo mais acentuado nos pacientes com idade superior a 70 anos de vida e do sexo masculino. A Sociedade Brasileira de Nefrologia (SBN) no censo de 2017 aponta que a pessoa se torna mais propensa a desenvolver a doença entre 45 a 64 anos de vida. Pacheco, Dell Agnolo, Magnani e Hungare (2012) reafirma estes dados determinando como doença da idade, com perda progressiva dos nefros a medida que a pessoa envelhece, sendo determinada esta, como patologia inerente ao próprio envelhecimento humano, contudo o declínio da função renal pode ser acelerado por fatores modificáveis como, consumo indiscriminado de medicamentos, não controle da pressão arterial, diabetes e dislipidemia.

Conforme Schmidt et al. (2016), 70\% dos pacientes admitidos na UTI desenvolveram LRA, que além de outros fatores já relatados que corroboram para incidência desta patologia, existem outras causas de grande relevância como a sepse sendo a principal causa, ventilação mecânica, uso prolongado de anti-inflamatório não asteroidais, insuficiência respiratória, doenças cardiovasculares, acidente vascular encefálico e infecção do trato urinário.

Pacheco (2012) chama atenção para as drogas/fármacos que são utilizados em pacientes críticos, uma vez que são altamente nefrotóxicas, pois quase sempre se faz necessário para tratamento dos mesmos. O autor enfatiza tal importância em virtude do número de pacientes que desenvolveram LRA em sua pesquisa, onde 79\% faziam uso de drogas vasoativas, $34 \%$ de antibióticos, $34 \%$ de analgesia, $17 \%$ em uso de sedativo.

Neste sentido Grassi, Dell'Acqua, Jensen, Fontes e Guimarães. (2017), ratifica com seu estudo que o choque séptico é um dos fatores responsáveis com 37\% de sua amostragem, o qual contribuiu para o surgimento, evolução e gravidade da LRA, os sedativos com 54\%, $61 \%$ em uso de droga vasoativa e $68 \%$ dos pacientes eram submetido a ventilação mecânica.

Barbosa et al. (2012) trouxe em seu trabalho que dos 22 pacientes internados na UTI que desenvolveram LRA durante o período de internação, $32 \%$ fazia tratamento com antibioticoterapia, $32 \%$ com analgésico, sedativo $18 \%$, drogas vasoativas 22\%, antitrombolítico 77\%. Em estudo realizado por Souza (2010), quanto a patologias pré-existentes: diabéticos 59\%, hipertensos 54\%, dislipidêmicos $27 \%$, tabagistas $63 \%$; deste, $45 \%$ desenvolveram LRA. 
Assim pode-se observar que esta patologia é decorrente de inúmeros fatores independentemente do tipo ou estágio, dentre as causas o envelhecimento e, por conseguinte os fatores aos quais são submetidos quando internados (medicamentos/ procedimentos). Desta forma vale destacar a atuação da enfermagem como um dos atores principais neste cenário, uma vez que é o profissional enfermeiro quem fica diariamente atuando na assistência ininterruptamente em prol da evolução do quadro clínico e evitando intercorrências preveníveis.

Estudo desenvolvido por Guedes, Silva, Carvalho e Oliveira (2017) traz que a incidência de LRA nos pacientes internados nas UTI é de $44,7 \%$, já outras literaturas trouxeram dados variados, porém semelhantes. Lima, Caseiro e Gagliane (2016) 43,5\%, Luft et al. (2016) 27\% e Ponche et al. (2011) 25,5\%. A mortalidade neste público também tem alta incidência respectivamente, $68 \%, 75,5 \%, 74 \%$ e $62 \%$. Assim pode ser constatado uma variabilidade de casos na literatura de alta incidência tanto de LRA quanto de óbitos.

Nesta perspectiva constatou-se que a LRA é uma etiologia multifatorial com elevada incidência em ambientes críticos, como a UTI, muitos eventos são resultantes de necrose tubular aguda, em decorrência de acontecimentos isquêmicos ou nefrotóxicos devido uso prolongado de diversos medicamentos, assim como procedimentos invasivos. A incidência de desfecho desfavorável e/ou mortalidade desses pacientes permanece alta, embora haja progresso das técnicas dialíticas e tecnologias avançada voltada para este público alvo. Diante dos elevados números de casos de LRA e óbito evidenciam-se a urgência de medidas preditivas, diagnóstico precoce e terapêutica efetiva.

Neste seguimento quando ocorre a LRA independente da causa, tipo, estágio, idade ou sexo se, diagnosticado e tratado precocemente ocorrerá a recuperação da função renal. Porém na maioria dos casos necessita-se de terapia dialítica para filtrar o sangue até que os rins retomem sua função. Nesta consonância, os pacientes que desenvolve LRA irão submeter-se a terapia dialítica, para reestabelecer o equilíbrio hidroeletrolítico e hemodinâmico.

A seleção da modalidade do tratamento advém do quadro clínico do paciente e da disponibilidade de cada instituição, desta forma a hemodiálise (HD) é o método mais utilizado por ser o tipo de terapia mais disponível e acessível nas instituições. Tal método é proposto/eleito após avaliação primorosa do paciente e análise dos exames laboratoriais, em prol de reestabelecer a função dos rins e permitir uma nova situação de equilíbrio para o mesmo.

A HD consiste no processo de filtragem sanguínea mediante uma máquina que sucede através de um filtro/dialisador por onde passa o sangue do paciente para serem retiradas as substâncias em excesso, o mesmo faz o trabalho que o rim comprometido não consegue. Este procedimento remove do organismo os resíduos prejudiciais à saúde, extraindo as impurezas, excesso de líquidos e substâncias indesejáveis, em seguida o sangue é devolvido ao paciente através de um acesso vascular apropriado, deste modo propicia o restabelecimento das condições clínica do mesmo (SBN, 2012).

Com o avanço tecnológico dos últimos anos o tratamento de HD tornou-se cada vez mais seguro e diversificado, proporcionando um tratamento mais individualizado no que se refere a prescrição de modalidades dialíticas, com isso é possível ter uma HD apropriada para o paciente, permitindo desta forma a escolha do tratamento conforme as condições clínicas do mesmo, e ainda, contribuindo para maior estabilidade hemodinâmica durante a sessão de hemodiálise e minimizando as intercorrências, o que contribuirá para menor tempo de internação, menores custos financeiro e maior qualidade de vida ao paciente.

Foi constando em estudo de Loiola Neto, Soares e Gonçalves (2017) que mesmo com as novas tecnologias em equipamentos para HD, 30\% das sessões de hemodiálise manifestam algum tipo de intercorrência em virtude deste tipo de terapia.

Diante do que foi exposto nota-se que o paciente renal em hemodiálise possui quadro clínico instável não só pelo próprio tratamento, mas pelo problema primário e suas comorbidades. Neste cenário, o enfermeiro atua prevenindo intercorrências, através da observância dos sinais, sintomas e queixas, intervindo nas alterações e possibilitando melhora no 
bem-estar do paciente em busca de minorar os desconfortos, possibilitando ainda readaptação imediata à sessão de HD. Ademais, a intervenção de enfermagem faz parte do processo do cuidado ao paciente, colaborando no controle dos temores, intervindo e avaliando as reações do doente diante das medidas adotadas e executadas e se necessário modificando-as, visando a melhora do quadro clínico e possibilitando a cura do paciente.

Segundo Resolução $n^{\circ} 358$ (2009), a SAE é uma metodologia utilizada no processo de trabalho que possibilita ao enfermeiro prestar uma assistência de enfermagem integral e humanizada, colocando em prática seus conhecimentos técnico, científicos e humanos no exercício profissional, seja ele no ambiente público ou privado o que traz respaldo cientifico ao profissional enfermeiro através do processo de enfermagem e registro adequado da assistência prestada.

Neste sentido, o profissional enfermeiro faz-se presente e atuante diante deste cenário, realizando e fazendo ser executada a SAE com a prescrição de enfermagem, inclusive registro rigoroso do balanço hídrico para quantificar a diurese, já que a mesma faz parte do critério para diagnóstico. Desta forma, sua implantação possibilita ao enfermeiro uma assistência com mais qualidade, eficiência e conferindo maior segurança aos pacientes e profissionais, pois esta ferramenta auxilia na organização do processo de trabalho evidenciando a contribuição da enfermagem na atenção à saúde.

As atribuições do enfermeiro perpassam a técnicas ou procedimentos bem realizados e eficientes, já que lançam mão de uma ação cuidativa integral, holística e contínua, além de uma efetiva comunicação enfermeiro-paciente (Santana, Fontenelle \& Magalhães, 2013).

De acordo com Cerqueira, Tavares e Machado (2014) são inúmeras as incumbências do enfermeiro que presta cuidados ao paciente portador de LRA em HD, este profissional atua diante das intercorrências intradialíticas, prevenindo possíveis complicações como: hipotensão/hipertensão arterial, hipoglicemia/hiperglicemia, desconforto respiratório, taquicardia, saturação, sangramentos, controle das perdas durante a HD, administração de medicamentos prescritos e ainda no tratamento de emergência das disfunções hidroeletrolíticas, avaliando a evolução e a resposta do paciente ao tratamento.

Diante disto a enfermagem trabalha oferecendo suporte físico e emocional, além de atuar cuidando do distúrbio primário que desencadeou a LRA. Quanto aos sinais clínicos e intercorrências apresentados durante a sessão de HD precisa ser atendido imediatamente de forma precisa e adequada para não se obtenha desfecho negativo, como piora do quadro clínico, prolongamento de permanência hospitalar, cronificação da doença ou até mesmo o óbito.

Nesta conjuntura o enfermeiro é um profissional habilitado e qualificado que desempenha função fundamental no acompanhamento do paciente em hemodiálise, pois ele é quem assiste diretamente o paciente durante a sessão de hemodiálise e é crucial que o mesmo atenda prontamente as potencias intercorrências. A assistência de enfermagem ao paciente em HD engloba o processo de enfermagem sistematizando, os cuidados de forma humanizada e individualizada mesmo antes do início do tratamento, sempre observando continuamente o aspecto geral e promovendo uma avaliação criteriosa (Silva, Marini \& Silva, 2016).

Nesta perspectiva se torna indispensável o papel do enfermeiro, pois este profissional apresenta conhecimento técnico-cientifico para atuar frente aos pacientes em hemodiálise e é o profissional que está mais próximo do paciente, desenvolvendo uma relação de confiança que contribui para colaboração do paciente no seu processo de recuperação e cura. $\mathrm{O}$ cuidado de enfermagem ao paciente portador da LRA em HD inclui a sistematização desde o início até o termino da sessão de hemodiálise, avaliando e monitorando o estado hemodinâmico initerruptamente através de um olhar acurado em prol de prevenção de instabilidade durante e pós-tratamento.

\section{Considerações Finais}

Percebeu-se que a LRA é uma patologia multifatorial e que pode ser antecipada por alguns achados. Independente de como a LRA seja classificada, o paciente apresentará desequilíbrio ácido-básico e hidroeletrolítico, impactando negativamente 
na evolução do quadro clínico e bem estar do mesmo, o que leva a uma instabilidade hemodinâmica, podendo até mesmo ir a óbito pela gravidade da doença. Nesta perspectiva, torna-se indispensável o papel do enfermeiro quando se discute o processo saúde doença, pois é um profissional capacitado, habilitado e que possui conhecimento técnico-cientifico e raciocínio crítico reflexivo nas tomadas de decisões.

Diante do exposto, o enfermeiro possui importante papel no cuidado ao paciente com LRA, uma vez que é imprescindível a atuação deste profissional para um tratamento efetivo, reduzindo assim as intercorrências durante a sessão de hemodiálise, o que contribui para a redução do tempo de internação nas UTIs e reestabelecimento das funções orgânicas o quanto antes, minimizando problemas de saúde futuros e otimizando a qualidade de vida deste paciente quando reinserido na sociedade.

No entanto, vale ressaltar que ainda existem lacunas na literatura sobre a importância da atuação do enfermeiro enquanto membro da equipe multidisciplinar no que tange a construção de um plano de cuidados para os pacientes dialíticos. Sendo esta uma fonte de futuros pesquisas na área.

\section{Referências}

Barbosa, K. R. A., Silva, S. C., Pope, S., Fornari, J. V., Rodrigues, F. S. M., Barnabé, A. S., \& Ferraz, R. R. N. (2012). Indicações de hemodiálise de emergência em uma unidade de terapia intensiva de um hospital particular da cidade de Atibaia - SP. Science in Health, 3(3), 131-138. http://arquivos.cruzeirodosuleducacional.edu.br/principal/new/revista_scienceinhealth/09_set_dez_2012/science_03_03_131-138.pdf

Barros, L. C. N., Silveira, F. S., Silveira, M. S., Morais, T. C., Nunes, M. A. P., \& Bastos, K. A. (2012). Insuficiência renal aguda em pacientes internados por insuficiência cardíaca descompensada. J Bras Nefrol., 34(2),122-129. http://www.scielo.br/scielo.php?script=sci_arttext\&pid=S0101-28002012000200004.

Cerqueira, D. P., Tavares, J. R., \& Machado, R. C. (2014). Fatores preditivos da insuficiência renal e algoritmo de controle e tratamento. Rev. Latino-Am. Enfermagem, 22(2), 211-217. https://www.revistas.usp.br/rlae/article/view/85054.

Grassi, M. F., Dell'Acqua, M. C. Q., Jensen, R., Fontes, C. M. B., \& Guimarães, H. C. Q. C. P. (2017). Diagnósticos, resultados e intervenções de enfermagem em pacientes com lesão renal aguda. Acta Paul Enferm., 30(5), 538-545. https://www.scielo.br/j/ape/a/yZd6jnPcmGKCSbJTtgkxDvw/?format=pdf.

Guedes, J. R., Silva, E. S., Carvalho, I. L. N., \& Oliveira, M. D. (2017). Incidência e fatores predisponentes de insuficiência renal aguda em unidade de terapia intensiva. Cogitare Enferm, 22 (2), e49035. https://revistas.ufpr.br/cogitare/article/view/49035.

Li P. K. T., Burdmann, E. A., \& Mehta. R. L. (2013). Injúria Renal Aguda: um alerta global. J Bras Nefrol., 35(1), 1-5. https://bjnephrology.org/article/injuriarenal-aguda-um-alerta-global/.

Lima, H. M. P., Caseiro, M. M., \& Gagliani, L. H. (2017). Principais fatores de internação do paciente com insuficiência renal aguda em unidade de terapia intensiva. Rev Soc Bras Clin Med., 15(2), 99-102. http://www.sbcm.org.br/ojs3/index.php/rsbcm/article/download/266/248/.

Luft, J., Boes, A. A., Lazzari, D. D., Nascimento, E. R. P., Busana, J. A., \& Canever, B. P. (2016). Lesão renal aguda em unidade de tratamento intensivo: características clínicas e desfechos. Cogitare Enferm., 21(2), 01-09. https://revistas.ufpr.br/cogitare/article/view/43822.

Loiola Neto, I. R., Soares, G. L., \& Gonçalves, A. S. (2017). O papel do enfermeiro de uma unidade de terapia intensiva na hemodiálise. UNINGÁ Review, 31(1) 40-44. https://www.mastereditora.com.br/periodico/20170801_220045.pdf

Marconi, M. A. \& Lakatos, E. M. (2017). Fundamentos de metodologia científica. (8a ed). Atlas.

Nunes, T. F., Brunetta, D. M., Leal, C. M., Pisi, P. C. B., \& Roris Filho, J. S. (2010). Insuficiência renal aguda. Medicina (Ribeirão Preto), 43(3), 272-282. https://www.revistas.usp.br/rmrp/article/view/184.

Pacheco, A., Dell Agnolo, C., Magnani, A., \& Hungare, J. (2012). Insuficiência renal aguda em hospital ensino. Revista UNINGÁ, 34(1). http://ec2-34-233-57254.compute-1.amazonaws.com/index.php/uninga/article/view/1075

Padilha, K. G., Vattimo, M. F. F., Silva, S. C., \& Kimura, M. (2010). Enfermagem em UTI: cuidado do paciente crítico. Monole.

Pares, L. A. B., Wandeur, V., \& Matsuo, T. (2015). Preditores de injúria renal aguda e de mortalidade em uma Unidade de Terapia Intensiva. J Bras Nefrol., 37(1), 38-46. https://www.scielo.br/scielo.php?script=sci_abstract\&pid=S0101-28002015000100038\&lng=pt\&tlng=pt

Resolução $n^{o}$ 7, de 24 de fevereiro de 2010. (2010). Dispõe sobre os requisitos mínimos para funcionamento de Unidades de Terapia Intensiva e dá outras providências.. http://bvsms.saude.gov.br/bvs/saudelegis/anvisa/2010/res0007_24_02_2010.html.

Resolução $n^{o}$ 358, de 15 de outubro de 2009. (2009). Dispõe sobre a sistematização da assistência de enfermagem e a implementação do processo de enfermagem em ambientes, públicos ou privados, em que ocorre o cuidado profissional de enfermagem. http://www.cofen.gov.br/resoluo-COFEN3582009_4384.html.

Santana, S. S., Fontenelle, T., \& Magalhães, L. M. (2013). Assistência de enfermagem prestada aos pacientes em Tratamento hemodialítico nas unidades de nefrologia. Rev. Científica do ITPAC, 6(3). https://assets.unitpac.com.br/arquivos/Revista/63/5.pdf 
Research, Society and Development, v. 10, n. 8, e12910817108, 2021

(CC BY 4.0) | ISSN 2525-3409 | DOI: http://dx.doi.org/10.33448/rsd-v10i8.17108

Schmidt, L., Wiese, L. P. L., Pereira, E. M., Possamai, K. S., Santos, E., \& Fernandes, F. M. (2016). Lesão renal aguda em pacientes críticos: perfil clínico e relação com processos infecciosos graves. Rev. Bras. Farm. Hosp. Serv. Saúde São Paulo, 7(3), 19-24. http://v1.sbrafh.org.br/public/artigos/2016070303000956BR.pdf.

Schor, N, Durão Junior, M. S, \& Kirsztajn, G. M. (2017). Lesão renal aguda: manual prático. Balieiro.

Silva, C. M. S., Silva, D. A. N., Silva, G. G. P., Maia, L. F. S., \& Oliveira, T. S. (2016). Insuficiência renal aguda: principais causas e a intervenção de enfermagem em UTI. Revista Recien, 6(16), 48-56. https://www.recien.com.br/index.php/Recien/article/download/134/pdf

Silva, M. S., Marini, T. S. O., \& Silva. C. F. B. (2016). Enfermagem e suas intervenções nas principais complicações ocorridas durante a sessão de hemodiálise. Rev. Enfermagem. e Saúde Coletiva, 1(2), 45-60. http://revesc.org/index.php/revesc/article/viewFile/7/9

Smeltzer, S. C., \& Bare, B. G. (2014). Brunner e Suddarth: Tratado de Enfermagem Médico-cirúrgica. (11a ed). Guanabara Koogan.

Sociedade Brasileira de Nefrologia (SBN). (2012). Minidicionário do paciente renal: terapia de substituição renal. Abbott. http://docplayer.com.br/71605Do-paciente-renal-terapia-de-substituicao-renal.html.

Sousa, V. J. (2015). A importância dos cuidados de enfermagem prestados em terapia intensiva a pacientes em processos hemodialíticos venovenosos contínuos. Rev. Eletrôn. Atualiza Saúde, 1(1), 99-108. http://atualizarevista.com.br/wp-content/uploads/2014/10/a-importancia-dos-cuidados-de-enfermagemprestados-em-terapia-intensiva-a-pacientes-em-processos-hemodialiticos-venovenosos-continuos-pesquisa-bibliografica-revista-atualiza-saude-n1-v1.pdf.

Souza, M. L., Malagutti, W., Rodrigues, F. S. M., Barnabé, A. S., Francisco, L., Silva, R. N., Deus, R. B., \& Ferraz, R. R. N. (2010). Incidência de insuficiência renal aguda e crônica como complicações de pacientes internados em uma unidade de terapia intensiva. ConScientiae Saúde, 9(3), 456-461. https://periodicos.uninove.br/saude/article/view/2165. 